\title{
Model Predictive Control for Linear Impulsive Systems
}

\author{
Technical Report 2012-01, Process Control \& Informatics Unit, \\ School of Chemical Engineering, NTU Athens \\ Pantelis Sopasakis ${ }^{1}$, Panagiotis Patrinos ${ }^{2}$, Haralambos Sarimveis ${ }^{1}$ and Alberto Bemporad ${ }^{2}$
}

\begin{abstract}
Linear Impulsive Control Systems have been extensively studied with respect to their equilibrium points which, in most cases, are no other than the origin. However, the trajectory of the system cannot be stabilized to arbitrary desired points which imposes a significant restriction towards their utilization in various applications such as drug administration.

In this paper, we study the equilibrium of Linear Impulsive Systems in light of target-sets instead of the standard equilibrium point approach. We properly extend the notion of invariant sets which is crucial in designing asymptotically stable Model Predictive Controllers (MPC).
\end{abstract}

\section{INTRODUCTION}

The motivation for this work comes mainly from the field of pharmacokinetics and the need for prescribing optimal and individualized drug administration policies. PhysiologicallyBased Pharmacokinetic (PBPK) models have been found to provide a reliable modeling framework for drug absorption, distribution, metabolism and elimination and there is already a lot of relevant experimental data available in the literature [1]. When the drug is administered orally or in any other way not continuously, instantaneous jumps are observed in the concentration of the drug in some organs; this is mathematically conceptualized as a discontinuity of the first kind and gives rise to the so called Impulsive Systems [2]. PBPK models are actually systems of differential equations and it is straightforward to cast them as impulsive differential systems [3].

Other systems have also been modeled using this approach. For instance, in [4] a model of a spacecraft is formulated as a linear impulsive system. Shen et al. use impulsive differential equations to describe the dynamics of a fed-batch fermentator [5]. However, there is a dearth in bibliographical references to applications of impulsive systems mainly due to the shortcomings of the current theoretical tools for their analysis and design.

Linear Impulsive Systems have been studied to a great extent regarding existence and uniqueness of solutions, stability and other qualitative properties [2], [6]. The existing theory tackles stability in light of the equilibrium points of the system and in most cases boils down to the study of the properties of the zero solution exclusively [7], [8]. For instance, Fontes and Pereira [9] prescribed stability conditions for the design of MPC for nonlinear impulsive

\footnotetext{
${ }^{1}$ School of Chemical Engineering, National Technical University of Athens, 9 Heroon Polytechneiou Street, 15780 Zografou Campus, Athens, Greece

${ }^{2}$ IMT Institute for Advanced Studies Lucca, Piazza S. Ponziano, 6, 55100 Lucca, Italy
}

systems using Nagumo-like invariance conditions, but without the guarantee that the intersample trajectory of the system satisfies the state constraints.

Except for trivial cases (e.g. $\dot{x}=0$ ), it is impossible to stabilize the state of an impulsive system at any given desired state - a fact that waxes the wealth of results in this field not applicable to a great load of scenarios of significant practical interest such as drug administration control. This calls for weaker stability qualifications such as stability with respect to a given target-set as opposed to the traditional approach that makes use of equilibrium points. This necessary generalization paves the way for the formulation and solution of MPC problems with linear impulsive models.

\section{PREliminaries AND Notation}

Consider the following linear impulsive system:

$$
\begin{aligned}
\frac{\mathrm{d} x}{\mathrm{~d} t} & =A x, \text { for } t \in \mathbb{R} \backslash T \mathbb{N} \\
\Delta x(k T) & =B u_{k}, k \in \mathbb{N}
\end{aligned}
$$

Where $x \in \mathbb{R}^{n}, u \in \mathbb{R}^{m}$ and $A$ and $B$ are matrices of proper dimensions. Often, we use the notation $\tau_{k}:=k T$ to denote the impulsive time instants. It should be clear that $T \mathbb{N}=$ $\{k T ; k \in \mathbb{N}\}$ where $T>0$ is a constant impulsive period. In (1b) we have used the notation $\Delta x(k T):=x\left(k T^{+}\right)-$ $x(k T)$ where $x\left(\tau_{k}^{+}\right):=\lim _{\xi \rightarrow \tau_{k}^{+}} x(\xi)$.

The system is subject to the following state and input constraints:

$$
x(t) \in \mathcal{X}, \forall t \geq 0, u_{k} \in \mathcal{U}, \forall k \in \mathbb{N}
$$

where $\mathcal{X}$ and $\mathcal{U}$ are assumed to be polyhedral sets.

Requirement (2) renders any optimization problem generated thereupon (as finite-horizon optimal control problems arising in MPC) semi-infinite since it employs an infinite number of constraints. Such a problem would be particularly difficult to cope with per se, so, we propose a methodology to convert these constraints into a finite set of affine inequalities. Such a reduction was proposed by Pierce and Schumitzky for a planar linear impulsive system of the Kruger-Thiemer form wherein $A$ has only real eigenvalues and the state and input constraints are assumed to be rectangular [3]. To overcome this crux, we employ polytopic inclusions of the continuoustime trajectory of the system as in our previous work on sampled-data systems with random time delay [10].

Let $\pi$ denote a sequence of inputs $\pi=$ $\left\{u_{0}, u_{1}, \ldots, u_{N-1}\right\}$ drawn from $\mathcal{U}$ and $\varphi\left(t ; x_{0}, \pi\right)$ be a solution of (1) satisfying $\varphi\left(0 ; x_{0}, \pi\right)=x_{0}$. Whenever 
we need to explicitly note that the initial time instant is other than 0 , we use the notation $\varphi\left(t ; \tau_{0}, x_{0}, \pi\right)$. Let $t>0$ and $\tau_{j}$ be the largest impulse time not exceeding $t$ and $j \leq N-1$. Then, for $\tau_{j}<t<\tau_{j+1}$ it is $\varphi\left(t ; x_{0}, \pi\right)=e^{A\left(t-\tau_{j}\right)} \varphi\left(\tau_{j}^{+} ; x_{0}, \pi\right)$, or, what is the same:

$$
\varphi\left(t ; x_{0}, \pi\right)=e^{A\left(t-\tau_{j}\right)}\left(e^{j A T} x_{0}+\sum_{i=0}^{j} e^{(j-i) A T} B u_{i}\right)
$$

In what follows, stability is considered with respect to a convex target-set $\mathcal{Z}$ into which we want to lead the state of the system. This set need not contain the origin.

Consider the following impulsive system $\Sigma^{g}$ in closedloop form:

$$
\begin{aligned}
\frac{\mathrm{d} x}{\mathrm{~d} t} & =A x, \text { for } t \in \mathbb{R} \backslash T \mathbb{N} \\
\Delta x\left(\tau_{k}\right) & =B g\left(x\left(\tau_{k}\right)\right), \text { for } k \in \mathbb{N}
\end{aligned}
$$

Let us denote by $\varphi_{c l}\left(t ; x_{0}, g(\cdot)\right)$ the closed-loop trajectory of the above system satisfying the initial condition $\varphi_{c l}\left(\tau_{0} ; x_{0}, g(\cdot)\right)=x_{0}$. Hereinafter we shall use the notation co $\{\Gamma\}$ to denote the convex hull of a set $\Gamma$, that is:

$$
\operatorname{co}\{\Gamma\}=\left\{\begin{array}{l|l}
z=\sum_{i=1}^{n} \alpha_{i} \gamma_{i} \mid \begin{array}{c}
\forall i \in \mathbb{N}_{[1, n]}: \gamma_{i} \in \Gamma, \\
\alpha_{i} \in[0,1], \sum_{i=1}^{n} \alpha_{i}=1
\end{array}
\end{array}\right\}
$$

and the notation cone $\{\Gamma\}$ to denote the conic hull of $\Gamma$ :

$$
\text { cone }\{\Gamma\}=\left\{\begin{array}{l|l}
z=\sum_{i=1}^{n} \beta_{i} \gamma_{i} & \begin{array}{c}
\forall i \in \mathbb{N}_{[1, n]}: \\
\beta_{i} \geq 0, \gamma_{i} \in \Gamma
\end{array}
\end{array}\right\}
$$

Whenever $\Gamma$ is finite, the corresponding convex or conic hull is said to be finitely-generated. Recall that any polytope can be represented as the convex hull of a finite set of vectors and every polyhedron $\mathcal{H}$ can be represented as $\mathcal{H}=\mathcal{P}+\mathcal{C}$ where $\mathcal{P}$ is a polytope and $\mathcal{C}$ is a finitely-generated cone.

\section{Controlled InVARiant Sets}

Given a set $\mathcal{Z} \subseteq \mathcal{X}$, which stands as a convex targetset we need to determine a set $\mathcal{Y} \subseteq \mathcal{Z}$ such that for every $y \in \mathcal{Y}$ there is a $u \in \mathcal{U}$ so that the following conditions are simultaneously satisfied:

A1. $\varphi(T ; y, u) \in \mathcal{Y}$, where $\varphi(T ; y, u)=\Phi(T)(y+B u)$ and where $\Phi(t):=e^{A t}$.

A2. $\mathcal{W}(y, u):=\operatorname{cl}\{\varphi(r ; y, u) ; r \in(0, T]\} \subseteq \mathcal{Z}$, where cl stands for the set closure.

In what follows, the set $\mathcal{Z}$ is assumed to be polyhedral, i.e. $\mathcal{Z}=\left\{z \in \mathbb{R}^{n} \mid H_{z} z \leq K_{z}\right\}$ For a given state $y$ and input $u$, we construct a polytope $S(y, u)$ such that

$$
S(y, u) \supseteq \operatorname{cl}\{\varphi(r ; y, u) ; r \in(0, T]\}
$$

Thus, introducing some conservatism, we may replace A2 by:

$$
\text { A3. } S(y, u) \subseteq \mathcal{Z}
$$

and notice that condition A3 implies condition A2. The set $\mathcal{Y}$ will be hereinafter referred to as impulsively controlled invariant with respect to $\mathcal{Z}$. Once the state enters $\mathcal{Y}$, it will remain in $\mathcal{Z}$ at all impulsive and continuous time instants and after time $T$ will return to $\mathcal{Y}$. The foregoing discussion gives rise to the following definitions:

Definition 1 (Impulsively Controlled Invariant Set): Given a set $\mathcal{Z}$, a set $\mathcal{Y} \subseteq \mathcal{Z}$ such that for every $y \in \mathcal{Y}$ there is a $u \in \mathcal{U}$ so that $\mathrm{A} 1$ and $\mathrm{A} 2$ hold true for the system (1a, $1 b)$ is called an impulsively controlled invariant set with respect to $\mathcal{Z}$.

Definition 2 (Impulsively Invariant Set): Consider the closed-loop impulsive system (4). Given a set $\mathcal{Z}$, a set $\mathcal{Y}$ such that for every $y \in \mathcal{Y}$, one has $\mathcal{W}(y, g(y)) \subseteq \mathcal{Z}$ and A1 holds is called an impulsively invariant set with respect to $\mathcal{Z}$.

A set $\mathcal{Y}$ satisfying conditions $\mathrm{A} 1$ and $\mathrm{A} 3$ has the property:

$$
\mathcal{Y} \subseteq \Theta^{S}(\mathcal{Y})
$$

where

$$
\Theta^{S}(\mathcal{Y}):=\left\{\begin{array}{l|l}
y \in \mathbb{R}^{n} & \begin{array}{l}
\exists u \in \mathcal{U} \text { s.t. } S(y, u) \subseteq \mathcal{Z} \\
\text { and } \Phi(T)(y+B u) \in \mathcal{Y}
\end{array}
\end{array}\right\}
$$

In what follows, we shall focus on those sets $\mathcal{Y}$ that are fixed points of the operator $\Theta^{S}$, i.e. they satisfy (6) with equality $\left(\mathcal{Y}=\Theta^{S}(\mathcal{Y})\right)$. This choice actually provides a computational procedure for the calculation of such sets on which we will elaborate in what follows.

The set $S(x, u)$, being a polytope, can be written as the convex hull of its extreme points. Let $\left\{A_{i}\right\}_{i=1}^{K}$ be a collection of matrices so that $\left\{e^{A t} ; t \in[0, T]\right\} \subseteq \operatorname{co}\left\{A_{i}\right\}_{i=1}^{K}$. Such a collection can be determined by methods of polytopic overapproximation of the matrix exponential function as in [10]. Then,

$$
S(y, u)=(y+B u) \cdot \operatorname{co}\left\{A_{i}\right\}_{i \in \mathbb{N}_{[1, K]}}
$$

Hence, the set $\mathcal{Y}$ can be restated as follows using the projection operator $\pi_{x}: \mathcal{X} \times \mathcal{U} \ni(x, u) \mapsto \pi_{x}(x, u)=$ $x \in \mathcal{X}$ :

$$
\mathcal{Y}=\pi_{x}\left\{(y, u) \in \mathbb{R}^{n+m} \mid \begin{array}{c}
\Phi(T)(y+B u) \in \mathcal{Y} \\
A_{i}(y+B u) \in \mathcal{Z} ; i \in \mathbb{N}_{[1, K]}
\end{array}\right\}
$$

The set $\mathcal{Y}$ is the fixed point of the mapping:

$$
F(\Omega):=\pi_{x}\left\{(y, u) \mid \begin{array}{c}
\Phi(T)(y+B u) \in \Omega \\
A_{i}(y+B u) \in \mathcal{Z} ; i \in \mathbb{N}_{[1, K]}
\end{array}\right\},
$$

i.e., $\mathcal{Y}=F(\mathcal{Y})$, it can be therefore computed using a standard fixed-point iteration as is outlined below.

If Algorithm 1 converges in a finite number of steps to a nonempty set, then the resulting set $\mathcal{Y}$ is impulsively controlled invariant and polyhedral, i.e., it admits an $\mathcal{H}$ representation $\mathcal{Y}=\left\{y \in \mathbb{R}^{n}, H_{y} y \leq K_{y}\right\}$.

Having computed the set $\mathcal{Y}$, it is expedient to compute the set Pre $(\mathcal{Y})$ of all states that can be steered in one step inside $\mathcal{Y}$ while the continuous time trajectory between the two successive impulse times remains in $\mathcal{X}$. This is defined as:

$$
\operatorname{Pre}(\mathcal{Y})=\left\{\begin{array}{l|l}
x \in \mathcal{X} & \begin{array}{c}
\exists u \in \mathcal{U}, \Phi(T)(x+B u) \in \mathcal{Y} \\
S(x, u) \subseteq \mathcal{X}
\end{array}
\end{array}\right\}
$$

We may successively define the sets $\operatorname{Pre}^{k+1}(\mathcal{Y})=$ $\operatorname{Pre}\left(\operatorname{Pre}^{k}(\mathcal{Y})\right)$ for $k \in \mathbb{N}$ with $\operatorname{Pre}^{0}(\mathcal{Y})=\mathcal{Y}$. The set 


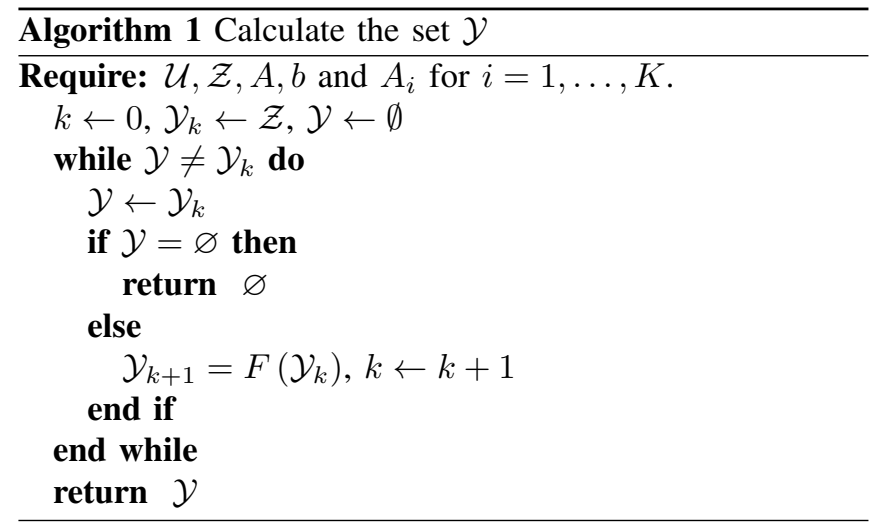

$\operatorname{Pre}^{k}(\mathcal{Y})$ contains all initial states at some impulse time that can be led inside $\mathcal{Y}$ in $k$ steps or less using a sequence of inputs $\pi=\left\{u_{j}\right\}_{k \in \mathbb{N}_{[0, k-1]}}$.

To this end we provide a criterion for a polyhedron to be impulsively controlled invariant with respect to a given set $\mathcal{Z}$.

Proposition 1: Let $\mathcal{Y}$ be a polyhedron and $\mathcal{Z}$ a convex set such that $\mathcal{Y} \subseteq \mathcal{Z}$ and assume that $\mathcal{Y}=\operatorname{co}\left\{y_{i}\right\}_{i \in \mathbb{N}_{[1, M]}}+$ cone $\left\{y_{j}\right\}_{j \in \mathbb{N}_{[M+1, P]}}$. Then, if for every $i \in \mathbb{N}_{[1, P]}$, there is a $u_{i} \in \mathcal{U}$ such that $\Phi(T)\left(y_{i}+B u_{i}\right) \in \mathcal{Y}$ while $S\left(y_{i}, u_{i}\right) \subseteq$ $\mathcal{Z}$, then $\mathcal{Y}$ is an impulsively controlled invariant set with respect to $\mathcal{Z}$.

Proof: For every $y \in \mathcal{Y}$ there are $\left\{\alpha_{j}(y)\right\}_{j \in \mathbb{N}_{[1, P]}}$ with $\alpha_{j} \geq 0$ and $\sum_{j=1}^{M} \alpha_{j}=1$ such that $y=\sum_{j=1}^{P} \alpha_{j} y_{j}$. Let $u=\sum_{j=1}^{P} \alpha_{j} u_{j}$. Then,

$$
\Phi(T)(y+B u)=\sum_{j=1}^{P} \alpha_{j} \Phi(T)\left(y_{j}+B u_{j}\right)
$$

Thus, $\Phi(T)(y+B u) \in \mathcal{Y}$ since $\mathcal{Y}$ is convex. Additionally, $S\left(y_{j}, u_{j}\right) \subseteq \mathcal{Z}$ is equivalent to the set of constraints $A_{i}\left(y_{j}+B u_{j}\right) \in \mathcal{Z}$ for all $j \in \mathbb{N}_{[1, P]}$ and $i \in \mathbb{N}_{[1, K]}$. Then,

$$
A_{i}(y+B u)=\sum_{i=1}^{P} \alpha_{j} A_{i}\left(y_{j}+B u_{j}\right)
$$

And since $\mathcal{Z}$ is convex and for all $j \in \mathbb{N}_{[M+1, P]}$ and $\lambda \geq$ $0, \lambda y_{j} \in \mathcal{Z}$, we have $A_{i}(y+B u) \in \mathcal{Z}$. Therefore, $\mathcal{Y}$ is impulsively controlled invariant with respect to $\mathcal{Z}$.

In this paper, invariance is studied in light of a targetset $\mathcal{Z}$; it is therefore different from the definition used in the paper of Pereira et al. where the traditional definition of invariant sets is employed [11]. The framework we introduce here is more flexible as it requires no elaborate properties for the target-set $\mathcal{Z}$ except for being polyhedral. Second, it harmonizes with control practice where the target-set is a predefined requirement as in drug administration (therapeutic window, [12]).

In the following section we properly extend the notion of stability of sets in light of the newly introduced impulsively controlled invariant sets with respect to given target-sets.

\section{Stability of Sets}

Consider the closed-loop impulsive system $\Sigma^{g}$ described by (4). In this section we introduce new definitions for stability of sets.

Definition 3 (Stable Sets): A nonempty set $\mathcal{Z} \subseteq \mathcal{X}$ is said to be stable for an impulsive system $\Sigma^{g}$ described by (4) with respect to a nonempty set $\mathcal{Y}$ if $\forall \varepsilon>0, \exists \delta>0$

$$
\operatorname{dist}_{\mathcal{Z}}\left(\varphi_{c l}\left(t ; 0, x_{0}, g(\cdot)\right)\right)<\varepsilon, \forall t \geq 0
$$

whenever $\operatorname{dist} \mathcal{Y}\left(x_{0}\right)<\delta$, where $\operatorname{dist}_{\mathcal{Z}}(x):=$ $\min _{z \in \mathcal{Z}}\|x-z\|$. The set $\mathcal{Z}$ is said to be an (locally) asymptotically stable set for $\Sigma^{g}$ with respect to $\mathcal{Y}$ if additionally there is an $\varepsilon_{0}>0$ such that $\lim _{t \rightarrow \infty} \operatorname{dist}_{\mathcal{Z}}\left(\varphi_{c l}\left(t ; x_{0}, g(\cdot)\right)\right)=0$ for all $x_{0}$ with $\operatorname{dist} y\left(x_{0}\right)<\varepsilon_{0}$.

Definition 4 (Domain of Attraction): If $\mathcal{Z}$ is stable for $\Sigma^{g}$ with respect to $\mathcal{Y}$ and for all $x_{0} \in A$ it is

$$
\lim _{t \rightarrow \infty} \operatorname{dist}_{\mathcal{Z}}\left(\varphi_{c l}\left(t ; x_{0}, g(\cdot)\right)\right)=0
$$

then we say that $\mathcal{Z}$ is asymptotically stable with respect to $\mathcal{Y}$ and $A$ is its domain of attraction.

This definition of stability is weaker than the one introduced by Bainov and Stamova in [13] in the sense that if a set $\mathcal{Z}$ is stable with respect to a given set $\mathcal{Y}$, it need not be a stable set unless $\mathcal{Y} \supseteq \mathcal{Z}$.

Definition 5 (Weakly Stable Sets): A set $\mathcal{Z}$ is said to be weakly stable for the impulsive system $\Sigma^{g}$ - as in (4) - with respect to $\mathcal{Y}$ if

$$
\begin{gathered}
\forall \varepsilon>0, \exists \delta>0, \operatorname{dist}_{\mathcal{Y}}\left(x_{0}\right)<\delta \Rightarrow \\
\operatorname{dist}_{\mathcal{Z}}\left(\varphi_{c l}\left(\tau_{k} ; 0, x_{0}, g(\cdot)\right)\right)<\varepsilon, \forall k \in \mathbb{N}
\end{gathered}
$$

Furthermore, $\mathcal{Z}$ is said to be weakly asymptotically stable if it is weakly stable and there is a $\varepsilon_{0}>0$ so that:

$$
\lim _{k \rightarrow \infty} \operatorname{dist}_{\mathcal{Z}}\left(\varphi_{c l}\left(\tau_{k} ; 0, x_{0}, g(\cdot)\right)\right)=0
$$

whenever disty $\left(x_{0}\right)<\varepsilon_{0}$.

The domain of attraction for weakly asymptotically stable systems is defined analogously.

Results from systems theory involving $\mathcal{K}$-class and $\mathcal{K} \mathcal{L}$ class functions can be properly extended to describe stability of sets as defined above. This leads to the following proposition:

Proposition 2 (Characterization of stability of sets): Let $\mathcal{Z}$ and $\mathcal{Y}$ be nonempty sets. The following statements are equivalent:

1) $\mathcal{Z}$ is a stable set for (4) with respect to $\mathcal{Y}$

2) There is a $\mathcal{K}$-class function $\alpha$ and a constant $c>0$ such that $\operatorname{dist}_{\mathcal{Z}}\left(\varphi_{c l}\left(t, 0, x_{0}, g(\cdot)\right)\right) \leq \alpha\left(\operatorname{dist} y\left(x_{0}\right)\right)$ whenever $\operatorname{dist}_{y}\left(x_{0}\right) \leq c$, for all $t \geq 0$.

Proof: This result is adapted for impuslive systems from [14] where is it stated for continuous time LTI systems. The proof is analogous.

Proposition 3 (Consequence of stability): If $\mathcal{Z}$ is stable for $\Sigma^{g}$ with respect to $\mathcal{Y}$ then any trajectory starting inside $\operatorname{cl} \mathcal{Y}$ will remain inside $\operatorname{cl} \mathcal{Z}$. 
Proof: Since $\mathcal{Z}$ is stable with respect to $\Sigma^{g}$, there is a $c>0$ and an $\alpha \in \mathcal{K}$ so that $\operatorname{dist}_{\mathcal{Z}}\left(\varphi_{c l}\left(t, 0, x_{0}, g(\cdot)\right)\right) \leq$ $\alpha\left(\operatorname{dist} y\left(x_{0}\right)\right)$ for all $t \geq 0$ whenever $\operatorname{dist} y\left(x_{0}\right)<$ $c$. Let $x_{0} \in \operatorname{cl} \mathcal{Y}$. Then $\operatorname{dist} \mathcal{Y}\left(x_{0}\right)=0<c$ and $\operatorname{dist}_{\mathcal{Z}}\left(\varphi_{c l}\left(t, 0, x_{0}, g(\cdot)\right)\right) \leq \alpha(0)=0$ for all $t \geq 0$ which means that $\varphi_{c l}\left(t, 0, x_{0}, g(\cdot)\right) \in \operatorname{cl} \mathcal{Z}$ for all $t \geq 0$. This proves the assertion.

Proposition 4 (Characterization of Asymptotic Stability): Let $\mathcal{Z}$ and $\mathcal{Y}$ be nonempty sets. The following statements are equivalent:

1) $\mathcal{Z}$ is an asymptotically stable set for (4) with respect to $\mathcal{Y}$

2) There is a $\mathcal{K} \mathcal{L}$-class function $\beta$ and a constant $c>0$ such that $\operatorname{dist}_{\mathcal{Z}}\left(\varphi_{c l}\left(t, 0, x_{0}, g(\cdot)\right)\right) \leq$ $\beta\left(\operatorname{dist} y\left(x_{0}\right), t\right)$ whenever $\operatorname{dist} y\left(x_{0}\right) \leq c$, for all $t \geq 0$.

Proof: The proof follows the exact steps found in [14] if we replace the standard metric of $\mathbb{R}^{n}$ with the point-to-set distance defined above.

Definition 6 (Uniform Boundedness): The trajectories of $\Sigma^{g}$ are called $(\mathcal{Z}, \mathcal{Y})$-locally uniformly bounded over an interval $I \subseteq[0, \infty)$ if there is an $\eta>0$ and a $\mathcal{K}$ class function $\alpha$ such that the following holds for all $t \in I \operatorname{dist}_{\mathcal{Z}}\left(\varphi_{c l}\left(t ; 0, x_{0}, g(\cdot)\right)\right) \leq \alpha\left(\operatorname{dist}_{\mathcal{Y}}\left(x_{0}\right)\right)$ whenever $\operatorname{dist}_{\mathcal{y}}\left(x_{0}\right)<\eta$.

It is natural to ask under what conditions (imposed on $g$ ), are the trajectories of $\Sigma^{g}(\mathcal{Z}, \mathcal{Y})$-locally uniformly bounded.

Proposition 5 (Criterion for Uniform Boundedness): Let $h_{g}(x):=x+B g(x)$ and assume that there exist a constant $\eta>0$ and a function $\alpha \in \mathcal{K}$ so that:

$$
\operatorname{dist}_{\mathcal{Z}}\left(h_{g}(x)\right) \leq \alpha(\operatorname{dist} \mathcal{Y}(x))
$$

whenever $\operatorname{dist} y(x)<\eta$. Then the trajectories of $\Sigma^{g}$ are $(\mathcal{Z}, \mathcal{Y})$-locally uniformly bounded.

Proof: Let,

$$
M:=\sup _{t \in(0, T]} \sup _{x \notin \mathcal{Z}} \frac{\operatorname{dist}_{\mathcal{Z}}\left(e^{A t} x\right)}{\operatorname{dist}_{\mathcal{Z}}(x)} \in[0, \infty)
$$

Then, for $t \in(0, T]$, we have:

$$
\begin{aligned}
\operatorname{dist}_{\mathcal{Z}}\left(\varphi_{c l}\left(t ; 0, x_{0}, g(\cdot)\right)\right) & \leq M \operatorname{dist}_{\mathcal{Z}}\left(x_{0}+B g\left(x_{0}\right)\right) \\
& \leq \alpha\left(\operatorname{dist} \mathcal{Y}\left(x_{0}\right)\right)
\end{aligned}
$$

whenever disty $(x)<\eta$, which proves the assertion.

Remark: Unless $\mathcal{Z}$ is a linear subspace or a convex cone, we may not assume that the triangle inequality holds for $\operatorname{dist}_{\mathcal{Z}}$, so it is not easy to simplify the expression $\operatorname{dist}_{\mathcal{Z}}(h(x))=\operatorname{dist}_{\mathcal{Z}}(x+B g(x))$. In that special case, it holds that $\operatorname{dist}_{\mathcal{Z}}(h(x)) \leq \operatorname{dist}_{\mathcal{Z}}(x)+M_{B} \operatorname{dist}_{\mathcal{Z}}(g(x))$ where

$$
M_{B}:=\sup _{x \notin \mathcal{Z}} \frac{\operatorname{dist}_{\mathcal{Z}}(B x)}{\operatorname{dist}_{\mathcal{Z}}(x)}
$$

Furthermore, if $g$ can be found to be uniformly continuous in an open set that includes $\mathcal{Y}$ as a subset, then $h_{g}$ is also uniformly continuous and $h_{g}(\mathcal{Y}) \subseteq \mathcal{Z}$, so (16) holds true.

It turns out that weak asymptotic stability and uniform boundedness entail asymptotic stability for a set $\mathcal{Z}$ under certain additional conditions establishing this way a clear analogy between the following result and Theorem 2.27 in [15] for Sampled-data systems.

Assumption 1: We assume that $\varnothing \neq \mathcal{Y} \subseteq \mathcal{Z}, \mathcal{Y}$ is impulsively invariant with respect to $\mathcal{Z}$ and there is a constant $\eta>0$ and a function $\omega \in \mathcal{K}$ such that for all $k \in \mathbb{N}$ :

$$
\operatorname{dist} y\left(\varphi_{c l}\left(k T ; 0, x_{0}, g(\cdot)\right)\right) \leq \omega\left(\operatorname{dist} \mathcal{Y}\left(x_{0}\right)\right)
$$

whenever $\operatorname{dist}_{\mathcal{Y}}\left(x_{0}\right)<\eta$. That is, $\mathcal{Y}$ is weakly stable with respect to itself.

Theorem 1 (Criterion for Asymptotic Stability): Let $\mathcal{Z}, \mathcal{Y}$ be given nonempty sets and Assumption 1 is satisfied. Assume that $\mathcal{Z}$ is weakly asymptotically stable for $\Sigma^{g}$ with respect to $\mathcal{Y}$ and the trajectories of $\Sigma^{g}$ are $(\mathcal{Z}, \mathcal{Y})$-locally uniformly bounded over $(0, T]$. Then $\mathcal{Z}$ is asymptotically stable with respect to $\mathcal{Y}$.

Proof: Since $\mathcal{Z}$ is weakly asymptotically stable with respect to $\mathcal{Y}$, there is a $\eta^{s}>0$ and a $\beta \in \mathcal{K} \mathcal{L}$ such that, if $\operatorname{dist}_{\mathcal{Y}}\left(x_{0}\right)<\eta^{s}$ it is: $\operatorname{dist}_{\mathcal{Z}}\left(\varphi_{c l}\left(k T ; 0, x_{0}, g(\cdot)\right)\right) \leq$ $\beta\left(\operatorname{dist}_{\mathcal{Y}}\left(x_{0}\right), k T\right)$ for all $k \in \mathbb{N}$.

Since $\Sigma^{g}$ is $(\mathcal{Z}, \mathcal{Y})$-locally uniformly bounded over $(0, T]$, there is a positive constant $\eta^{b}>0$ and an $\alpha \in \mathcal{K}$ such that for all $t \in(0, T]$

$$
\operatorname{dist}_{\mathcal{Z}}\left(\varphi_{c l}\left(t ; 0, x_{0}, g(\cdot)\right)\right) \leq \alpha\left(\operatorname{dist} \mathcal{Y}\left(x_{0}\right)\right)
$$

whenever $\operatorname{dist} y\left(x_{0}\right)<\eta^{b}$. Also, since assumption 1 holds, there is a positive constant $\eta^{y}$ and a function $\omega \in \mathcal{K}$ so that for all $k \in \mathbb{N}$ :

$$
\operatorname{dist} y\left(\varphi_{c l}\left(k T ; 0, x_{0}, g(\cdot)\right)\right) \leq \omega\left(\operatorname{dist} \mathcal{Y}\left(x_{0}\right)\right)
$$

for all $x_{0}$ such that disty $\left(x_{0}\right)<\eta^{y}$. Let $\gamma_{0}(r)=\beta(r, 0)$ and let $\eta:=\min \left\{\eta^{s}, \gamma_{0}^{-1}\left(\eta^{b}\right), \omega^{-1}\left(\eta^{b}\right)\right\}$. Let $x_{0}$ be such that $\operatorname{dist}_{\mathcal{Y}}\left(x_{0}\right)<\eta$. Then, $\operatorname{dist}_{\mathcal{Z}}\left(\varphi_{c l}\left(k T ; 0, x_{0}, g(\cdot)\right)\right) \leq$ $\beta\left(\operatorname{dist} \mathcal{Y}\left(x_{0}\right), k T\right) \leq \eta^{b}$ for all $k \in \mathbb{N}$. Define $\hat{x}_{0}:=$ $\varphi_{c l}\left(k T ; 0, x_{0}, g(\cdot)\right)$. Additionally, because of (20),

$$
\begin{aligned}
\operatorname{dist} \mathcal{Y}\left(\hat{x}_{0}\right) & =\operatorname{dist} \mathcal{Y}\left(\varphi_{c l}\left(k T ; x_{0}, g(\cdot)\right)\right) \\
& \leq \omega\left(\operatorname{dist} \mathcal{Y}\left(\hat{x}_{0}\right)\right)<\eta^{b}
\end{aligned}
$$

Since $\operatorname{dist} y\left(\hat{x}_{0}\right)<\eta^{b}$, inequality (19) applies to $\hat{x}_{0}$; so for $\xi \in(k T,(k+1) T]:$

$$
\begin{aligned}
& \operatorname{dist}_{\mathcal{Z}}\left(\varphi_{c l}\left(\xi ; 0, \hat{x}_{0}, g(\cdot)\right)\right) \leq \alpha\left(\operatorname{dist} \mathcal{Y}\left(\hat{x}_{0}\right)\right) \\
& =\alpha\left(\operatorname{dist} \mathcal{Y}\left(\varphi_{c l}\left(k T ; 0, x_{0}, g(\cdot)\right)\right)\right) \\
& \leq \alpha\left(\beta\left(\operatorname{dist}_{\mathcal{Y}}\left(x_{0}\right), k T\right)\right)
\end{aligned}
$$

holds for all $k \in \mathbb{N}$. Define $\hat{\beta}(r, s):=\alpha(\beta(r, s))$. Then $\hat{\beta}$ is $\mathcal{K} \mathcal{L}$-class and by lemma 1 in [16], without loss of generality $\hat{\beta}$ can be assumed to be uniformly incrementally bounded, that is, there exists a positive constant $P>0$ such that $\hat{\beta}(r, k T) \leq P \hat{\beta}(r,(k+1) T), \forall r \geq 0, \forall k \in \mathbb{N}$ and since $\hat{\beta}$ is strictly decreasing in the second argument, for any $s \in$ $[0,1]$, it is $\hat{\beta}(r,(k+1) T) \leq \hat{\beta}(r,(k+s) T)$. So, for $t \geq 0$ let $k_{0}=k_{0}(t) \in \mathbb{N}$ be the maximum integer so that $k_{0} T \leq t$. Equation (22) yields,

$$
\begin{aligned}
& \operatorname{dist}_{\mathcal{Z}}\left(\varphi_{c l}\left(t ; 0, \hat{x}_{0}, g(\cdot)\right)\right) \leq \hat{\beta}\left(\operatorname{dist} \mathcal{Y}\left(x_{0}\right), k_{0} T\right) \\
& \leq P \hat{\beta}\left(\operatorname{dist} \mathcal{Y}\left(x_{0}\right), k_{0} T+t-k_{0} T\right) \\
& =P \hat{\beta}\left(\operatorname{dist} \mathcal{Y}\left(x_{0}\right), t\right)
\end{aligned}
$$


The function $\tilde{\beta}(r, t):=P \hat{\beta}(r, t)$ is $\mathcal{K} \mathcal{L}$-class and by proposition 4 the proof is complete.

Taking quite similar steps we can prove the following criterion stability:

Theorem 2 (Criterion for Stability): Let $\mathcal{Z}, \mathcal{Y}$ be given nonempty sets and assumption 1 is satisfied and the trajectories of $\Sigma^{g}$ are $(\mathcal{Z}, \mathcal{Y})$-locally uniformly bounded over $(0, T]$. Then $\mathcal{Z}$ is stable with respect to $\mathcal{Y}$.

\section{Model Predictive Control}

\section{A. Formulation}

Unlike other existing MPC formulations for linear and nonlinear continuous time dynamical systems, it is not possible to stabilize the state of an impulsive system of the form (1) at any other state except possibly for the origin. The replacement of the notion of a set point with that of a targetset appears to provide a flexible framework for applying MPC to such systems.

For each $x \in \mathcal{X}$ we define the set-valued mapping $\mathcal{U}_{f}$ : $\mathcal{X} \rightrightarrows \mathcal{U}$ as follows:

$$
\mathcal{U}_{f}(x)=\left\{\begin{array}{l|c}
u \in \mathcal{U} & \begin{array}{c}
\Phi(T)(x+B u) \in \mathcal{Y} \\
A_{i}(x+B u) \in \mathcal{Z} ; i \in \mathbb{N}_{[1, K]}
\end{array}
\end{array}\right\}
$$

Notice that if $x \notin \mathcal{Y}$ then and only then $\mathcal{U}_{f}(x)=\varnothing$, that is $\operatorname{dom} \mathcal{U}_{f}=\mathcal{Y}$. We also define the set $D$ as follows:

$$
D=\left\{(x, u) \in \mathbb{R}^{n+m} \mid x \in \mathcal{Y}, u \in \mathcal{U}_{f}(x)\right\}
$$

This set is the graph of the set-valued mapping $\mathcal{U}_{f}$. To this end, we introduce the following stage cost function:

$$
\ell(x, u)=\operatorname{dist}_{D}^{2}(x, u)=\min _{(z, v) \in D}\|(x, u)-(z, v)\|^{2}
$$

Notice that $\ell(x, u)=0$ if and only if $x \in \mathcal{Y}$ and $u \in \mathcal{U}_{f}(x)$. This stage cost will allow to automatically perform dualmode MPC without actually having computed an auxiliary, local controller beforehand.

The proposed MPC scheme amounts in solving at every impulse time $\tau_{k}$ the following finite horizon optimal control problem:

$$
V_{N}^{\star}\left(x\left(\tau_{k}\right)\right)=\inf _{\pi \in \mathcal{U}_{N}\left(x\left(\tau_{k}\right)\right)} V_{N}\left(x\left(\tau_{k}\right), \pi\right)
$$

where

$$
V_{N}\left(x\left(\tau_{k}\right), \pi\right)=\sum_{j=0}^{N-1} \ell\left(\varphi\left(\tau_{k+j} ; x\left(\tau_{k}\right), \pi\right), u_{j}\right)
$$

and

$$
\mathcal{U}_{N}(x)=\left\{\begin{array}{c}
\forall j \in \mathbb{N}_{[0, N-1]}: u_{j} \in \mathcal{U} \\
S\left(\varphi\left(\tau_{k+j} ; x, \pi\right), u_{j}\right) \subseteq \mathcal{X} \\
\varphi\left(\tau_{k+N-1} ; x, \pi\right) \in \mathcal{Y}
\end{array}\right\}
$$

The MPC problem (27) can be reformulated as a convex QP:

$$
V_{N}^{\star}\left(x\left(\tau_{k}\right)\right)=\inf _{\pi, z, v} \bar{V}_{N}\left(x\left(\tau_{k}\right), \pi, z, v\right)
$$

where

$$
\bar{V}_{N}(x, \pi, z, v):=\sum_{j=0}^{N-1}\left\|\left[\begin{array}{c}
\varphi\left(\tau_{k+j} ; x, \pi\right)-z_{j} \\
u_{j}-v_{j}
\end{array}\right]\right\|^{2}
$$

subject to the constraints:

$$
\pi \in \mathcal{U}_{N}\left(x\left(\tau_{k}\right)\right),\left(z_{j}, v_{j}\right) \in D, \forall j \in \mathbb{N}_{[0, N-1]}
$$

The feasible domain of this problem is simply the set $\mathcal{X}_{N}=\operatorname{Pre}^{N-1}(\mathcal{Y})$. This problem is merely convex, therefore the optimizer is in general set-valued. This is evident since for every $x \in \mathcal{Y}$ and every $u \in \mathcal{U}_{f}(x)$ it holds that $\ell(x, u)=0$. Let us denote by $\pi^{\star}\left(x\left(\tau_{k}\right)\right)=$ $\left(\pi_{0}^{\star}\left(x\left(\tau_{k}\right)\right), \pi_{1}^{\star}\left(x\left(\tau_{k}\right)\right), \ldots, \pi_{N-1}^{\star}\left(x\left(\tau_{k}\right)\right)\right)$ the set-valued optimizer of (27). This gives rise to a family of feedback control laws $\sigma\left(x\left(\tau_{k}\right)\right)=\pi_{0}^{\star}\left(x\left(\tau_{k}\right)\right)$ and notice that if $x \in \mathcal{Y}$ then $\sigma(x)=\mathcal{U}_{f}(x)$. Then every $s: \mathcal{X}_{N} \rightarrow \mathcal{U}$ such that $s(x) \in \sigma(x)$ for all $x \in \mathcal{X}_{N}$ is called an optimal control law.

The following result is necessary to guarantee asymptotic stability of the closed-loop system with the MPC controller.

Proposition 6: For $x_{0} \in \mathcal{X}_{N} \backslash \mathcal{Y}, \sigma$ is single-valued and Lipschitz continuous.

Proof: The cost function is written as $V_{N}\left(x_{0}, \pi\right)=$ $\ell\left(x_{0}, u_{0}\right)+\vartheta\left(x_{0}, \bar{\pi}\right)$ where $\bar{\pi}$ is the trailling part of $\pi$ following $u_{0}$. One has that:

$$
x_{j}=\Phi(T)^{j} x_{0}+\sum_{p=0}^{j-1} \Phi(T)^{p+2} B u_{p}
$$

which we shall simply write as:

$$
x_{j}=L_{j} x_{0}+\sum_{p=1}^{N-1} Y_{p} u_{p}
$$

we then have that

$$
\vartheta\left(x_{0}, \bar{\pi}\right)=\sum_{j=1}^{N-1} \ell\left(L_{j} x_{0}+\sum_{p=1}^{N-1} Y_{p} u_{p}, u_{j}\right)
$$

$\ell\left(x_{0}, u_{0}\right)=\operatorname{dist}_{D}\left(x_{0}, u_{0}\right)$ is strictly convex for $x_{0} \in$ $\mathcal{X}_{N} \backslash \mathcal{Y}$ and $\vartheta$ is convex, so $V_{N}$ is strictly convex outside $\mathcal{Y}$. As a result the solution mapping $\sigma(\cdot)$ is single valued and Lipschitz continuous [17]. A fortiori it is uniformly continuous.

\section{B. Stability Properties}

Proposition 7 (Weak Stability of MPC and Invariance):

Given a target-set $\mathcal{Z}$ assume that there is a nonempty set $\mathcal{Y}$ which is impulsively controlled invariant with respect to $\mathcal{Z}$. Let $s: \mathcal{X}_{N} \rightarrow \mathcal{U}$ be an optimal control law for (27). Then, $\mathcal{Z}$ is weakly asymptotically stable with respect to $\mathcal{Y}$ with domain of attraction the set $\mathcal{X}_{N}$.

Proof: Step 1. We prove a Lyapunov-type inequality. First, we show that for all $x\left(\tau_{k}\right) \in \mathcal{X}_{N}$, it holds true that

$$
V_{N}^{\star}\left(x\left(\tau_{k+1}\right)\right)-V_{N}^{\star}\left(x\left(\tau_{k}\right)\right) \leq-\ell\left(x\left(\tau_{k}\right), s\left(x\left(\tau_{k}\right)\right)\right)
$$

where $x\left(\tau_{k+1}\right):=\varphi_{c l}\left(\tau_{k+1} ; \tau_{k}, x\left(\tau_{k}\right), s(\cdot)\right)=$ $\Phi(T)\left(x\left(\tau_{k}\right)+B s\left(x\left(\tau_{k}\right)\right)\right)$.

Equation (34) is an immediate consequence of the fact that if $\pi^{\star}\left(x\left(\tau_{k}\right)\right)$ is an optimal sequence of inputs and $\hat{\pi}^{\star}\left(x\left(\tau_{k}\right)\right) \in \pi^{\star}\left(x\left(\tau_{k}\right)\right)$ is an arbitrary optimal sequence, then for $u_{\tau_{k+N-1}}=\hat{\pi}_{N-1}^{\star}\left(x\left(\tau_{k}\right)\right)$ and $x_{\tau_{k+N-1}}=$ 
$\varphi_{c l}\left(\tau_{k+N-1} ; x\left(\tau_{k}\right), \hat{\pi}^{\star}\left(x\left(\tau_{k}\right)\right)\right)$ we have that $x_{\tau_{k+N-1}} \in \mathcal{Y}$ and $\ell\left(x_{\tau_{k+N-1}}, u_{\tau_{k+N-1}}\right)=0$. Indeed, let $x \in \mathcal{X}_{N}$ be an initial state at $\tau_{k}$. We denote by

$$
\pi^{\star}(x)=\left\{u_{k}^{\star}(x), u_{k+1}^{\star}(x), \ldots, u_{k+N-1}^{\star}(x)\right\}
$$

the optimal control sequence for which it holds that $V_{N}^{\star}(x)=$ $V_{N}\left(x, \pi^{\star}\right)$. If we apply $\pi^{\star}(x)$ to the impulsive system, the following sequence of states will occur:

$$
\mathbf{x}^{\star}(x)=\left\{x_{k}^{\star}(x), x_{k+1}^{\star}(x), \ldots, x_{k+N}^{\star}(x)\right\}
$$

where $x_{k}^{\star}(x)=x, x_{k+i}^{\star}(x)=\varphi_{c l}\left(\tau_{k+i} ; \tau_{k}, x, \pi^{\star}\right)$ for $i=$ $1, \ldots, N$, and $x_{k+N}^{\star}(x) \in \mathcal{Y}$. At the next time instant, $\tau_{k+1}$, the state of the system will be $x_{k+1}^{\star}(x)$ for which we chose the (sub-optimal) control sequence

$$
\tilde{\pi}(x)=\left\{u_{k+1}^{\star}(x), \ldots, u_{k+N-1}^{\star}(x), u\right\}
$$

where the last element $u$ is to be determined. The resulting sequence of states will be

$$
\begin{gathered}
\tilde{\mathbf{x}}(x)=\left\{x_{k+1}^{\star}(x), \ldots, x_{k+N}^{\star}(x),\right. \\
\left.\varphi_{c l}\left(\tau_{k+N+1} ; \tau_{k+N}, x_{k+N}^{\star}(x), u\right)\right\}
\end{gathered}
$$

Provided that $\varphi_{c l}\left(\tau_{k+N+1} ; \tau_{k+N}, x_{k+N}^{\star}(x), u\right) \in \mathcal{Y}$, the sequence of inputs $\tilde{\pi}(x)$ is admissible (but not necessarily optimal). This is accomplished by choosing $u \in \mathcal{U}_{f}\left(x_{k+N}^{\star}(x)\right) \neq$ $\varnothing$. We have that:

$$
V_{N}^{\star}(x)=V_{N}\left(x, \pi^{\star}(x)\right)=\sum_{j=0}^{N-1} \ell\left(x_{k+j}^{\star}(x), u_{k+j}^{\star}(x)\right)
$$

while

$$
\begin{aligned}
V_{N}\left(x_{k+1}^{\star}(x), \tilde{\pi}\right) & =V_{N}^{\star}(x)-\ell\left(x, u_{k}^{\star}(x)\right) \\
& +\ell\left(\varphi_{c l}\left(\tau_{k+N+1} ; \tau_{k+N}, x_{k+N}^{\star}(x), u\right), u\right) \\
& =V_{N}^{\star}(x)-\ell\left(x, u_{k}^{\star}(x)\right) \\
& =V_{N}^{\star}(x)-\ell(x, s(x))
\end{aligned}
$$

Because of the fact that $\tilde{\pi}(x)$ is sub-optimal, and making use of the previous equation, it is:

$$
\begin{aligned}
V_{N}^{\star}\left(x_{k+1}^{\star}(x)\right) & \leq V_{N}\left(x_{k+1}^{\star}(x), \tilde{\pi}\right) \\
& =V_{N}^{\star}(x)-\ell(x, s(x))
\end{aligned}
$$

for all $x \in \mathcal{X}_{N}$, which proves (34).

Step 2. Weak Stability of $\mathcal{Y}$. For $\varepsilon>0$, let us denote by $\mathcal{B}_{\varepsilon}^{\mathcal{Y}}:=\left\{x \in \mathcal{X}_{N}, \operatorname{dist}_{\mathcal{Y}}(x) \leq \varepsilon\right\}$. Let also

$$
\Omega_{\beta}:=\left\{x \in \mathcal{B}_{\varepsilon}^{\mathcal{Y}}, V_{N}^{\star}(x) \leq \beta\right\} .
$$

Take $x_{0} \in \Omega_{\beta} \subseteq \mathcal{B}_{\varepsilon}^{\mathcal{Y}}$. Then, be (34), one has that $V_{N}^{\star}\left(\varphi_{c l}\left(\tau_{k+1} ; \tau_{k}, x_{0}, s(\cdot)\right)\right) \leq V_{N}^{\star}\left(x_{0}\right) \leq \beta$, hence $\varphi_{c l}\left(\tau_{k+1} ; \tau_{k}, x_{0}, s(\cdot)\right) \in \Omega_{\beta}$. Since $V_{N}^{\star}$ takes the value 0 on $\mathcal{Y}$ and $\Omega_{\beta} \supseteq \mathcal{Y}$ and $V_{N}^{\star}$ is continuous, there is a $\eta>0$ such that $\mathcal{B}_{\eta}^{\mathcal{Y}} \subseteq \Omega_{\beta}$. So, if we choose $x_{0} \in \mathcal{B}_{\eta}^{\mathcal{Y}}$, that is $\operatorname{dist}_{\mathcal{Y}}\left(x_{0}\right)<\eta$, we have that $\varphi_{c l}\left(\tau_{k+1} ; \tau_{k}, x_{0}, s(\cdot)\right) \in \mathcal{B}_{\varepsilon}^{\mathcal{Y}}$ so $\mathcal{Y}$ is weakly stable with respect to itself.

Step 3. Asymptotic Stability and Attractivity of $\mathcal{Y}$ over $\mathcal{X}_{N}$. We now need to prove that

$$
\lim _{j \rightarrow \infty} \operatorname{dist} \mathcal{Y}\left(\varphi_{c l}\left(\tau_{k+j} ; \tau_{k}, x, s(\cdot)\right)\right)=0
$$

for all $x \in \mathcal{X}_{N} . V_{N}^{\star}$ is strictly decreasing and nonnegative outside $\mathcal{Y}$, so there is a $c \geq 0$ so that

$$
\lim _{j \rightarrow \infty} V_{N}^{\star}\left(\varphi_{c l}\left(\tau_{k+j} ; \tau_{k}, x, s(\cdot)\right)\right)=c
$$

Let

$$
\tilde{\Omega}_{c}=\left\{x \in \mathcal{X}_{N} \mid V_{N}^{\star}(x) \leq c\right\}
$$

Assume that $c>0$ and let $\zeta>0$ such that $\mathcal{B}_{\zeta}^{\mathcal{Y}} \subseteq \tilde{\Omega}_{c}$. Then, for all $j>0, V_{N}^{\star}\left(\varphi_{c l}\left(\tau_{k+j} ; \tau_{k}, x, s(\cdot)\right)\right)>\zeta$. Define $\gamma_{r}:=-\max _{\zeta \leq \operatorname{dist} y}(x) \leq r l(x, s(x))$ for $r>\zeta$ (Evidently $\gamma_{r}<0$ for all $r>\zeta$ ). Then, by (34) it is:

$$
\begin{aligned}
V_{N}^{\star}\left(x\left(\tau_{k+j}\right)\right) \leq & V_{N}^{\star}\left(x\left(\tau_{k}\right)\right)- \\
& \sum_{i=0}^{j-1} \ell\left(x\left(\tau_{k+i}\right), s\left(x\left(\tau_{k+1}\right)\right)\right) \\
< & V_{N}^{\star}\left(x\left(\tau_{k}\right)\right)-j \gamma_{r}
\end{aligned}
$$

Eventually, for $j \geq \gamma_{r}{ }^{-1} V_{N}^{\star}\left(x\left(\tau_{k}\right)\right)$ we have that $V_{N}^{\star}\left(x\left(\tau_{k+j}\right)\right)<0$ which is a contradiction. As a result:

$$
\lim _{j \rightarrow \infty} V_{N}^{\star}\left(\varphi_{c l}\left(\tau_{k+j} ; \tau_{k}, x, s(\cdot)\right)\right)=0
$$

for all $x \in \mathcal{X}_{N}$. The set $\mathcal{Y}$ is therefore weakly asymptotically stable with respect to itself with domain of attraction the set $\mathcal{X}_{N}$. As a result, since $\mathcal{Z} \supseteq \mathcal{Y}, \mathcal{Z}$ is weakly asymptotically stable with respect to $\mathcal{Y}$ with domain of attraction $\mathcal{X}_{N}$.

Remark 1: By Proposition 6, $\sigma(\cdot)$ is a single-valued control law outside $\mathcal{Y}$ and Lipschitz-continuous. As a result, it is uniformly continuous over $\mathcal{X}_{N} \backslash \mathcal{Y}$ and $h_{s}(x):=x+B s(x)$ is also uniformly continuous. This being the case, (16) holds true and the closed-loop system trajectories are $(\mathcal{Z}, \mathcal{Y})$ locally uniformly bounded. According to Theorem $1, \mathcal{Z}$ is locally asymptotically stable with respect to $\mathcal{Y}$ and because of Proposition 7 the domain of attraction of the closed-loop system is $\mathcal{X}_{N}$.

Remark 2: $\mathcal{X}_{N}$ is not invariant in continuous-time; that is if $x\left(\tau_{k}\right) \in \mathcal{X}_{N}$, then on one hand at impulsive time instants the trajectory of the closed-loop will remain in $\mathcal{X}_{N}$

$$
\varphi_{c l}\left(\tau_{k+j} ; \tau_{k}, x\left(\tau_{k}\right), s(\cdot)\right) \in \mathcal{X}_{N}, \forall j \in \mathbb{N}
$$

but on the other hand, this property is not implied for the intermediate time instants. There may be time instants $\hat{t} \in\left[\tau_{k}, \tau_{k+1}\right)$ so that $x\left(\tau_{k}\right) \in \mathcal{X}_{N}$ and $x\left(\tau_{k+1}\right) \in \mathcal{X}_{N}$, but $\varphi_{c l}\left(\hat{t} ; \tau_{k}, x\left(\tau_{k}\right), s(\cdot)\right) \in \mathcal{X} \backslash \mathcal{X}_{N}$. However, in the interim between impulsive time instants, the state trajectory will be bounded inside $\mathcal{X}$, thus the imposed constraints will not be violated. At the same time, $\mathcal{X}_{N}$ is a feasible subset of $\mathcal{X}$.

Remark 3: The set $\mathcal{Y}$ in Proposition 7 is impulsively invariant for the closed-loop system with respect to $\mathcal{Z}$. Indeed, if $x\left(\tau_{k}\right) \in \mathcal{Y}$, the the MPC feedback law possesses the property $s(x) \in \mathcal{U}_{f}(x)$ and by definition of $\mathcal{U}_{f}$ and since $\mathcal{Y}=\operatorname{dom} \mathcal{U}_{f}$, impulsive invariance follows. 


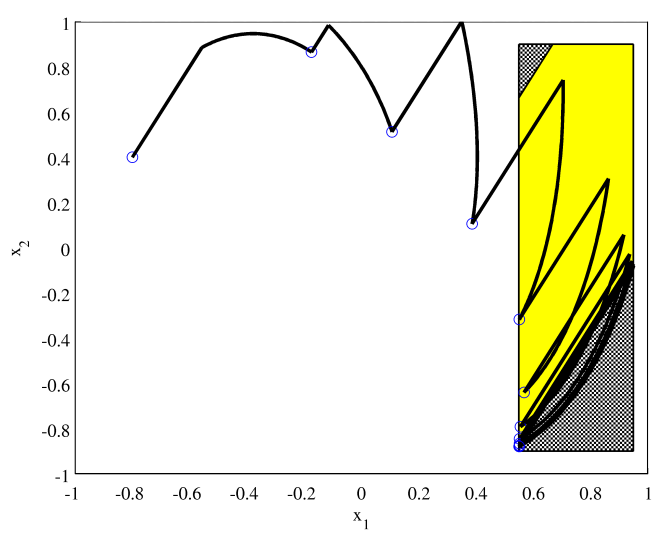

Fig. 1. The set of state constraints $\mathcal{X}$ (white), the target-set $\mathcal{Z}$ (checkboard) and the impulsively controlled invariant set $\mathcal{Y}$ (yellow). The continuous time trajectory of the system after an impulse is also illustrated starting from the initial point $x_{0}=[-0.8,0.4]$ and using the MPC feedback with prediction horizon $N=5$.

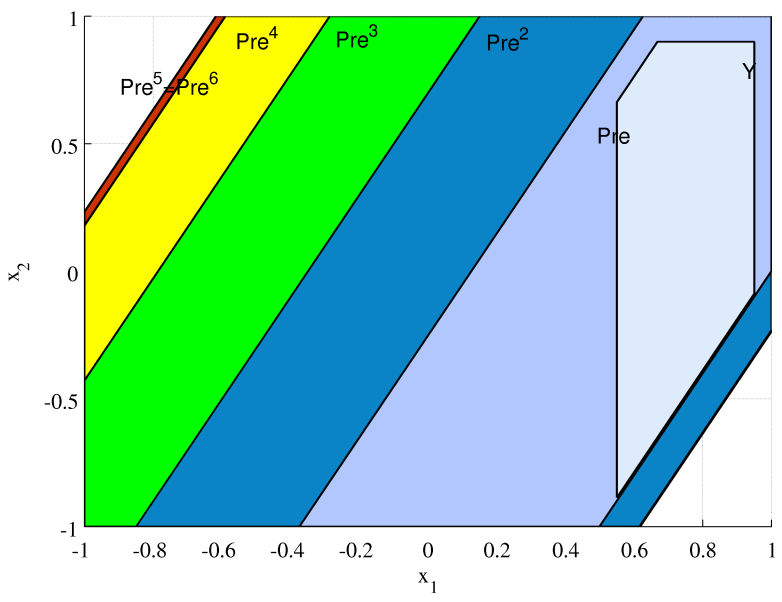

Fig. 2. The impuslively controlled invariant set $\mathcal{Y}$ and feasible regions for different prediction horizon values.

\section{Illustrative Example}

We provide here an illustrative example of the computation of the aforementioned impulsively controlled invariant set for the linear planar single-input impulsive system with matrices:

$$
A=\left[\begin{array}{ll}
-1 & 1 \\
-5 & 2
\end{array}\right] \text { and } B=\left[\begin{array}{l}
1 \\
2
\end{array}\right]
$$

and impulsive period $T=0.3$. The system is subject to the state and input constraints given by:

$$
\mathcal{X}=\mathbb{R}_{[-1,1]}^{2} \text { and } \mathcal{U}=[-1,1]
$$

We consider the following target-set which does not contain the origin:

$$
\mathcal{Z}=\left\{z \in \mathbb{R}^{n},\left[\begin{array}{c}
I_{2} \\
-I_{2}
\end{array}\right] z \leq\left[\begin{array}{c}
0.95 \\
0.9 \\
-0.55 \\
0.9
\end{array}\right]\right\}
$$

The set $\mathcal{Y}$ was computed using Algorithm 1 which terminated in 3 iterations. The three sets $\mathcal{X}, \mathcal{Z}$ and $\mathcal{Y}$ are illustrated in Fig. 1. An MPC controller was designed for this system using prediction horizon $N=6$. For every $x$ inside $\mathcal{Y}$, the controller picks a value from $\mathcal{U}_{f}(x)$ and applies it to the system - any such value assures that $\mathcal{Y}$ is impuslively controlled invariant with respect to $\mathcal{Z}$ for the closed-loop system. In this example the state enters the set $\mathcal{Y}$ so by definition it will stay in $\mathcal{Y}$ at all future impulse times and that whole continuous-time trajectory of the system will be bound to be inside $\mathcal{Z}$.

\section{ACKNOWLEDGMENT}

The work of the first author was financially supported by the NTUA Senator Committee of Basic Research Program $65 / 1867$. The work of the second and fourth author was partially supported by the HYCON2: Highly-complex and Networked Control Systems, Network of Excellence, FP7IST contract no. 257462 and by the European project EPRICE: Price-based Control of Electrical Power Systems, FP7-IST contract no. 249096.

\section{REFERENCES}

[1] R. Corley, Comprehensive Toxicology, vol. 12, ch. 12.04 Pharmacokinetics and PBPK models, pp. 27-58. Elsevier, 2010.

[2] T. Yang, Impulsive Control Theory, vol. 272 of Lecture Notes in Control and Information Sciences. Springer, 2001.

[3] J. G. Pierce and A. Schumitzky, "Optimal impulsive control of compartment models, i: Qualitative aspects," Journal of Optimization Theory and Applications, vol. 18, pp. 537-554, Apr. 1976.

[4] T. E. Carter, "Optimal impulsive space trajectories based on linear equations," Journal of Optimization Theory and Applications, vol. 70 pp. 277-297, Aug. 1991.

[5] L. Shen, Y. Wang, E. Feng, and Z. Xiu, "Bilevel parameters identification for the multi-stage nonlinear impulsive system in microorganisms fed-batch cultures," Nonlinear Analysis: Real World Applications, vol. 9, pp. 1068-1077, 2007.

[6] M. Benchohra, J. Henderson, and S. Ntouyas, Impulsive Differential Equations and Inclusions. Hindawi Publishing Corporation, 2006.

[7] A. M. Samoilenko and N. A. Perestyuk, Impulsive Differential Equations, vol. 14 of Nonlinear Science. World Scientific, 1995.

[8] X. Liu, Y. Liu, and K. L. Teo, "Stability analysis of impulsive control systems," Mathematical and Computer Modelling, vol. 37, no. 1, pp. 1357-1370, 2003.

[9] F. A. Fontes and F. L. Pereira, "Model predictive control of impulsive dynamical systems," in 4th IFAC NMPC, (The Netherlands), pp. 305310 , aug 2012.

[10] P. Patrinos, P. Sopasakis, and H. Sarimveis, "Stochastic model predictive control for constrained networked control systems with random time delay," in Proc. 18th IFAC World Congress, aug 28 - sep 22011.

[11] F. L. Pereira, G. N. Silva, and V. Oliveira, "Invariance for impulsive conrtol systems," Automation and Remote Control, vol. 69, no. 5, pp. 788-800, 2008

[12] J. Sun, Y. Li, W. Fang, and L. Mao, "Therapeutic time window for treatment of focal cerebral ischemia reperfusion injury with $\mathrm{xq}-1 \mathrm{~h}$ in rats," European Journal of Pharmacology, vol. 666, pp. 105-110, Sept. 2011.

[13] D. D. Bainov and I. M. Stamova, "Global stability of sets for impulsive differential-difference equations by luapunov's direct method," Acto Mathematica et Informatica Universitatis Ostraviensis, vol. 7, no. 1, pp. 7-22, 1999.

[14] H. K. Khalil, Nonlinear Systems. New Jersey: Prentice Hall, $3^{\text {rd }}$ ed., 2002.

[15] L. Grüne and J. Pannek, Nonlinear Model Predictive Control: Theory and Algorithms. Communications and Control Engineering, SpringerVerlag, 2011.

[16] D. Nešic, A. R. Teel, and E. D. Sontag, "On stability and input-tostate stability $\mathcal{K} \mathcal{L}$ estimates of discrete time and sampled data linear systems," in Proceedings of the American Control Conference, 1999.

[17] P. Patrinos and H. Sarimveis, "A new algorithm for solving convex parametric quadratic programs based on graphical derivatives of solution mappings," Automatica, vol. 46, pp. 1405-1418, 2010. 